\title{
Shifting an Object By Robot Using Haptic Shared Control System
}

\author{
Merline .G \\ ${ }^{1}$ Assistant Professor, Department of Electronics and communication Engineering, Jeppiaar Institute of Technology, Chennai, India
}

\begin{abstract}
A robot is used for the purpose of picking the object and placing it into another place. The robot setup is controlled by the android mobile with the blue-control application. Tele-manipulation allows human to perform operations in a remote environment. This system provides human operators with the ability to see, touch, and feel objects from a remote location. Haptic shared control is a promising approach to improve tele-manipulated task execution, by making safe and effective control actions tangible through guidance forces. The tele-manipulator serves as a tool to transfer movements from a human operator on a local station (the master) to a remote station (the slave), through a controller. Haptic shared control gives the visual information about the position, external and environmental forces acting on the robot to the human operator. Robot setup is used to pick and place the objects. There are many applications based on haptic shared control definition. Here, Tele operation of arm robot is implemented. This is used for the purpose of assembly in wireless manner controlled by human guidance. Also a wireless connection between remote and arm robot for picking and placing of objects.
\end{abstract}

Keywords: Robot, Tele-manipulation, Android, Haptic shared control

\section{Introduction}

In telemanipulation, a remote robot is controlled by a human and interacts with an environment while relaying information back to the human, providing access to environments which may be hostile, hazardous, or difficult to access. Shared control has the potential to overcome some of the limitations imposed by traditional telemanipulation architectures. Problems such as time delays and limitations in the fidelity of the master interface become less detrimental because commands from the operator are supplemented by local control. Shared control falls between supervisory control and bilateral control in that the human has the ability to control, and receive feedback from, the remote robot at a low level while maintaining the ability to supply high level commands .Under this control scheme, the human operator can intervene in an autonomous task executed by the robot and the robot can augment the direct commands generated by the operator.

\section{Literature Survey}

In H. Boessenkool et.al[1], Operator is assisted by forces applied at master device. It is hypothesized that continuous intuitive interaction between operator and support system will improve required time and accuracy with less control effort, even for imperfect transparency. An experimental study was performed in a hard-contact task environment. The subjects were aided by the designed shared control to perform a simple bolt-spanner task using a planar three degree of freedom (DOF) teleoperator. Haptic shared control was compared to normal operation for three levels of transparency. The experimental results showed that haptic shared control improves task performance, control effort and operator cognitive workload for the overall bolt-spanner task, for all three transparency levels. Analyses per subtask showed that free air movement (FAM) benefits most from shared control in terms of time performance, and also shows improved accuracy.
N. Stefanov et.al[2] - here the methodology is a computerassisted tele operation system. This paper introduces a computer-assisted teleoperation system, where the control over the teleoperator is shared between a human operator and computer assistance in order to improve the overall task performance. Two units, an action recognition and an assistance unit are introduced to provide context specific assistance. The action recognition unit can evaluate haptic data, handle high sampling rates, and deal with human behavior changes caused by the actived haptic assistance. Repairing of a broken hard drive is selected as scenario and three different task specific assistance functions are designed. The overall computer assisted teleoperation system is evaluated in two steps: first, the performance of the action recognition unit is evaluated and then, the performance of the integrated computer-assisted teleoperation system is compared with an unassisted system by means of a user study with 15 participants.

Ningbo Yu et.al[3] - the methodology used is Autonomous as well as teleoperated robots and wide applications in various environments. Their capability to accomplish complex and dynamic operations can be signi_cantly improved by fusing human intelligence with autonomous algorithms. In this paper, we propose a haptic shared control algorithm to provide _exible human assistance for semi-autonomous mobile robots. Through the admittance and impedance models, the haptic shared controller smoothly puts together human operator inputs with robot autonomy. Further, the level of autonomy is fully determined by the operator with the grasp motion. A decomposed design has been taken for the autonomous controller of the mobile robot. The algorithm was implemented on the haptic interface omega.7 together with a QBot mobile robot, and its feasibility and ef cacy have been validated by experiments.

J. Wildenbeest et.al[4] - the methodology used is teleoperation, haptic feedback allows the human operator to touch the remote environment. This paper presents a human 


\section{International Journal of Science and Research (IJSR) \\ ISSN (Online): 2319-7064}

Index Copernicus Value (2013): 6.14 | Impact Factor (2015): 6.391

factors experiment in which teleoperated task performance and control effort are assessed for a typical (dis-)assembly task in a hard-to-hard environment, well known to the operator. Subjects are provided with four levels of haptic feedback quality: no haptic feedback, low-frequency haptic feedback, combined low- and high-frequency haptic feedback, and the best possible - a natural spectrum of haptic feedback in a direct-controlled equivalent of the task. Four generalized fundamental subtasks are identified, namely: 1) free-space movement, 2) contact transition, 3) constrained translational, and 4) constrained rotational tasks. The results show that overall task performance and control effort are primarily improved by providing low-frequency haptic feedback (specifically by improvements in constrained translational and constrained rotational tasks), while further haptic feedback quality improvements yield only marginal performance increases and control effort decreases, even if a full natural spectrum of haptic feedback is provided.

Franck Mars et.al[5] - This study investigated humanmachine cooperation when driving with different degrees of a shared control system. By means of a direct intervention on the steering wheel, shared control systems partially correct the vehicle's trajectory and, at the same time, provide continuous haptic guidance to the driver. A crucial point is to determine the optimal level of steering assistance for effective cooperation between the two agents. Five system settings were compared with a condition in which no assistance was present. In addition, road visibility was manipulated by means of additional fog or self-controlled visual occlusions. Several performance indicators and subjective assessments were analyzed. The results show that the best repartition of control in terms of cooperation between human and machine can be identified through an analysis of the steering wheel reversal rate, the steering effort and the mean lateral position of the vehicle. The best cooperation was achieved with systems of relatively lowlevel haptic authority, although more intervention may be preferable in poor visibility conditions.

Henri Boessenkool ea.tl[6] - The experimental results provided evidence for the hypotheses, showing that the tested telemanipulation task benefits from haptic shared control, for three different levels of transparency. Essentially, the presence of haptic shared control allows for a worse transparency without compromising required time, and can even improve required time during perfect transparency.

Mark Mulder et.al[7] - Haptic feedback on the steering wheel is reported in literature as a promising way to support drivers during steering tasks. Haptic support allows drivers to remain in the direct manual control loop, avoiding known human factors issues with automation. The results indicated that continuous haptic guidance is a promising way to support drivers in actively producing (more) optimal steering actions during curve negotiation.

Paul Griffiths et.al[8] - In this paper, a paradigm for shared control is described in which a machine's manual control interface is motorized to allow a human and an automatic controller to simultaneously exert control. These results indicate that the haptic assist through the steering wheel improves lane keeping by at least $30 \%$ reduces visual demand by $29 \%(p<0.0001)$ and improves reaction time by $18 \mathrm{~ms}(\mathrm{p}=0.0009)$.

Haiying Hu et.al[9] - This paper describes a master-slave teleoperation system which is developed to evaluate the effectiveness of teleopresence in telerobotics applications. The operator wears a data glove augmented with an armgrounded force feedback device to control the dexterous hand and utilizes a Spaceball to control robot arm. Contact forces measured by the finger sensors can be feedback to the operator and visual telepresence systems collect the remote operation scenes and display to the operator by a stereo helmet.

Kenneth J. Waldron et.al[10] - A set of experiments was therefore designed to evaluate the efficacy of shared control for dexterous telemanipulation and to determine what combinations of force, visual and audio feedback provide the best performance and operator sense of presence. The results demonstrate the benefits of shared control and the need to choose carefully the types and methods of direct and indirect feedback.

\section{Block Diagram}

(1)

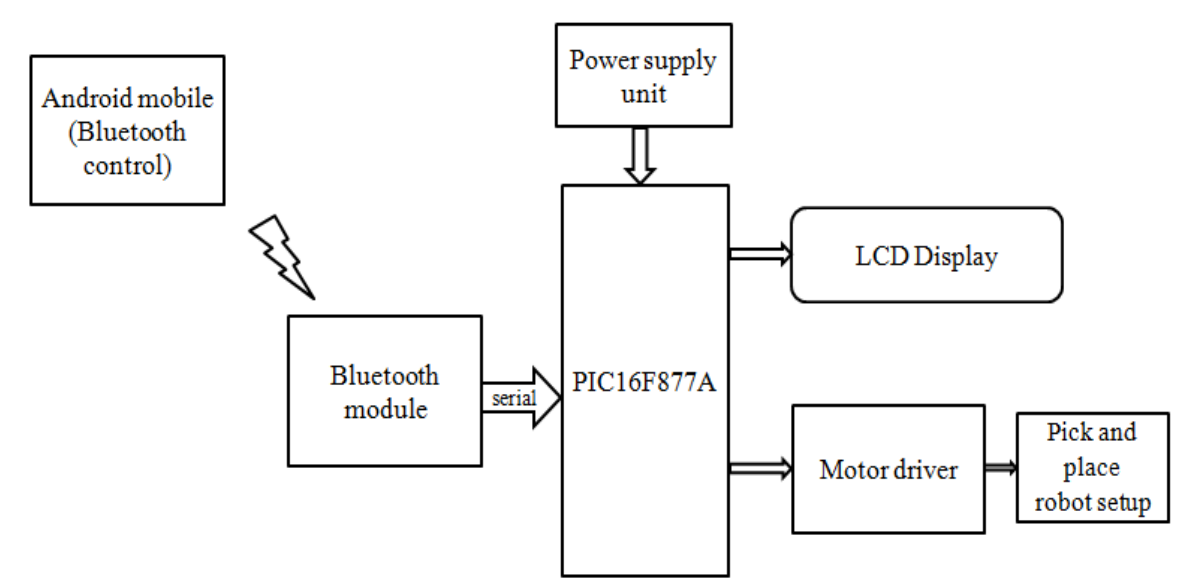

Figure 3.1: Block diagram of pick and place robot 


\section{International Journal of Science and Research (IJSR) \\ ISSN (Online): 2319-7064 \\ Index Copernicus Value (2013): 6.14 | Impact Factor (2015): 6.391}

Wireless connection between android mobile and the controller is established. In this setup the main components used are android application, blue tooth module, PIC controller, power supply, LCD display, motor driver circuit, robot setup. The android mobile is paired to the setup. Blue tooth module is serially communicated to the pic controller. The blue tooth module $\mathrm{HCO} 5$ is used to receive the data from the android mobile which contains blue control application. The power supply unit is used to convert the 230 ac to $5 \mathrm{v}$ dc. The microcontroller has $5 \mathrm{v}$ power supply and the motor has $12 \mathrm{v}$ power supply. The drive circuit is used to provide $12 \mathrm{v}$ power supply to the motor. The $16 \times 2 \mathrm{LCD}$ is used to display the command which is given by the human operator.

Here five motors are used for the commands-forward, reverse, left, right, up, down, pick and place. Two motors are connected in parallel for forward and reverse commands; rest three motors are connected to other commands respectively.

Robots can be classified into different categories depending on their function and the market needs they are designed for. Here we identify two major classes of robots, industrial robots and service robots. According to the Robotic Industries Association, an industrial robot is an automatically controlled, eprogrammable, multipurpose manipulator programmable in three or more axes which may be either fixed in place or mobile for use in industrial automation applications.

\section{Implementation and Performance Analysis}

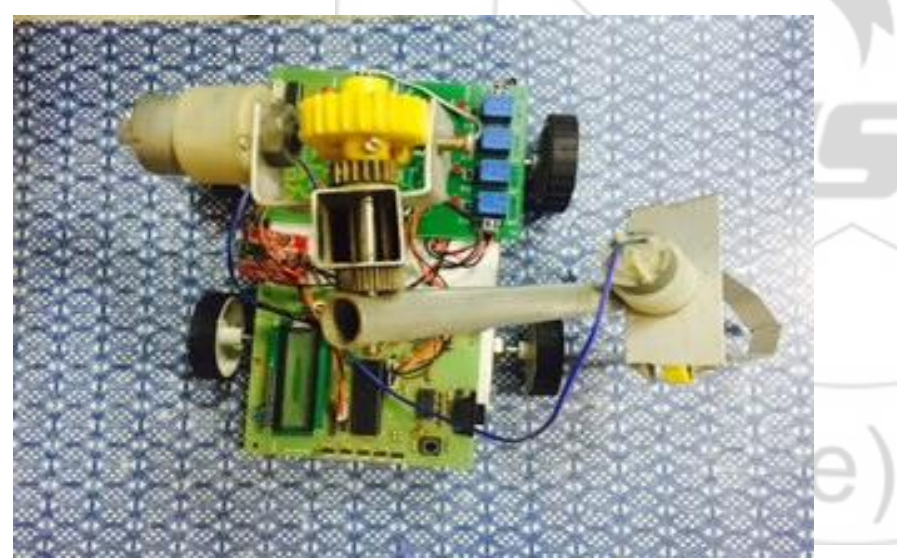

Figure 4.1: Side view of robot

Simulation is used when conducting experiments on a real system would be impossible or impractical. Here the software used is Proteus ISIS 7.7 which is a PCB design software integrated with the simulation of the circuit you design. It is integrated with real time simulation of the electronic circuit and test whether the designed circuit is working properly or not.

The program of microcontroller is loaded as .hex file. In this simulation result, the robot setup is displaying ANDROID APP BASED BLUE TOOTH ROBOT" when it is on. Then it displays ENTER YOUR CHOICE". The motors are controlled as per the user requirements. The motors operation taken by it is displayed on the LCD. The android application has the characters for the transmission of input actions given by the human operators.

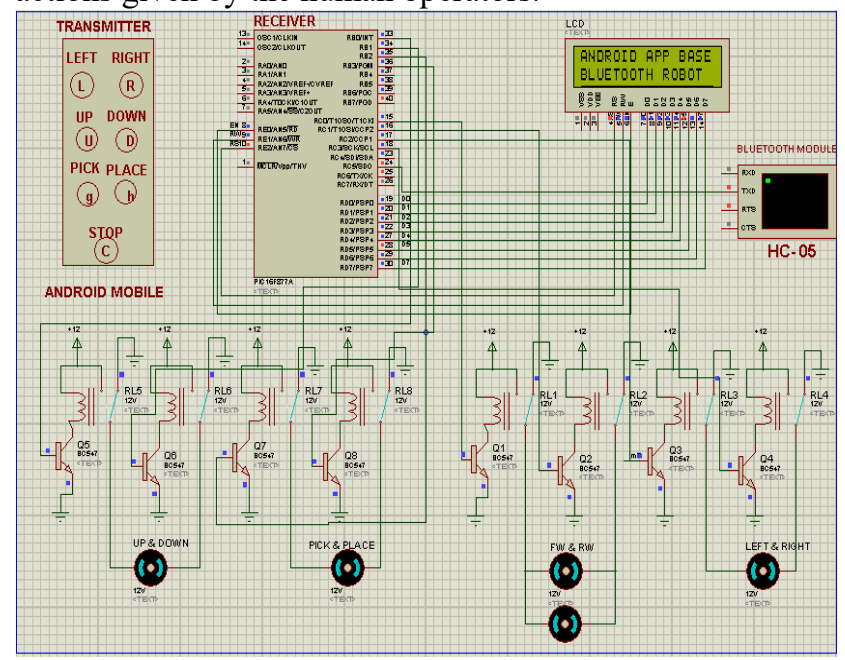

Figure 4.2: Initial on state

The first diagram shows the on-state position of the simulation it displays the android app base Bluetooth robot after some particular delay it displays the enter your choice

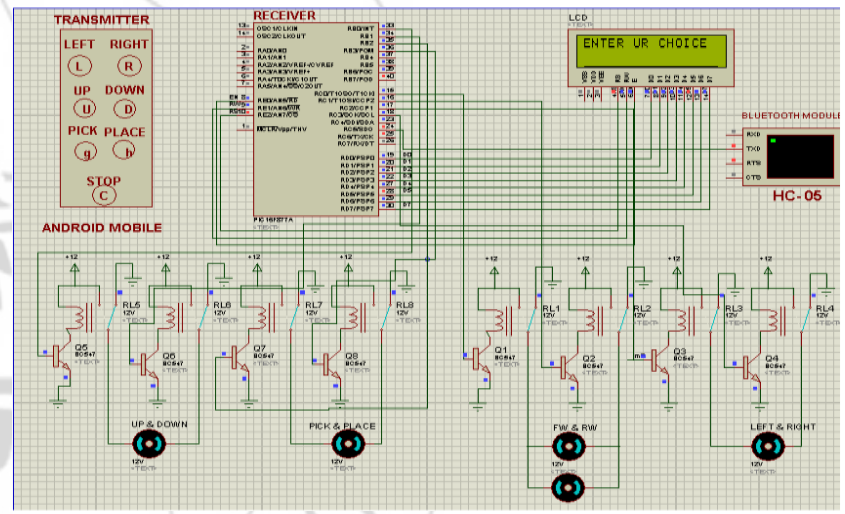

Figure 4.3: On state after some delay

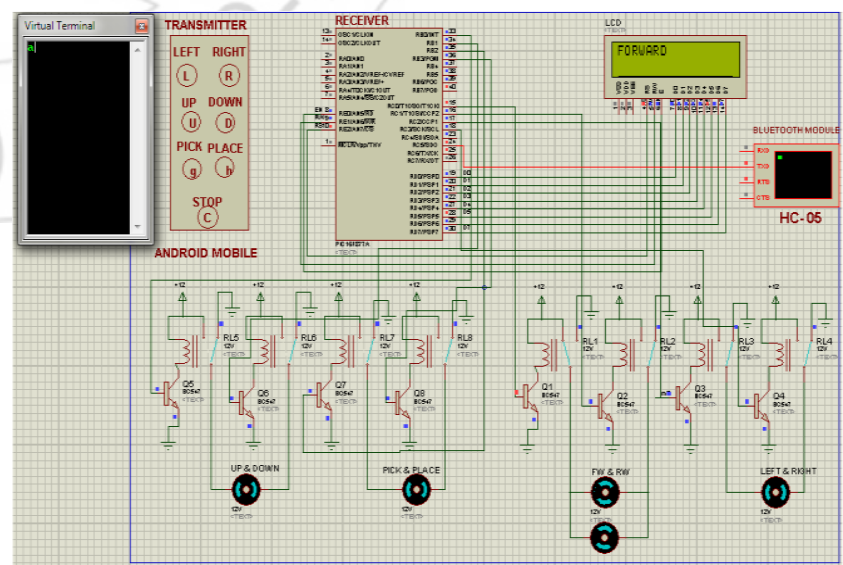

Figure 4.4: Forward Moving of robot

The button a" which is given in the virtual terminal describes the forward command where the motor rotates in the forward direction. Forward and reverse motor are connected in parallel manner. 


\section{International Journal of Science and Research (IJSR) \\ ISSN (Online): 2319-7064}

Index Copernicus Value (2013): 6.14 | Impact Factor (2015): 6.391

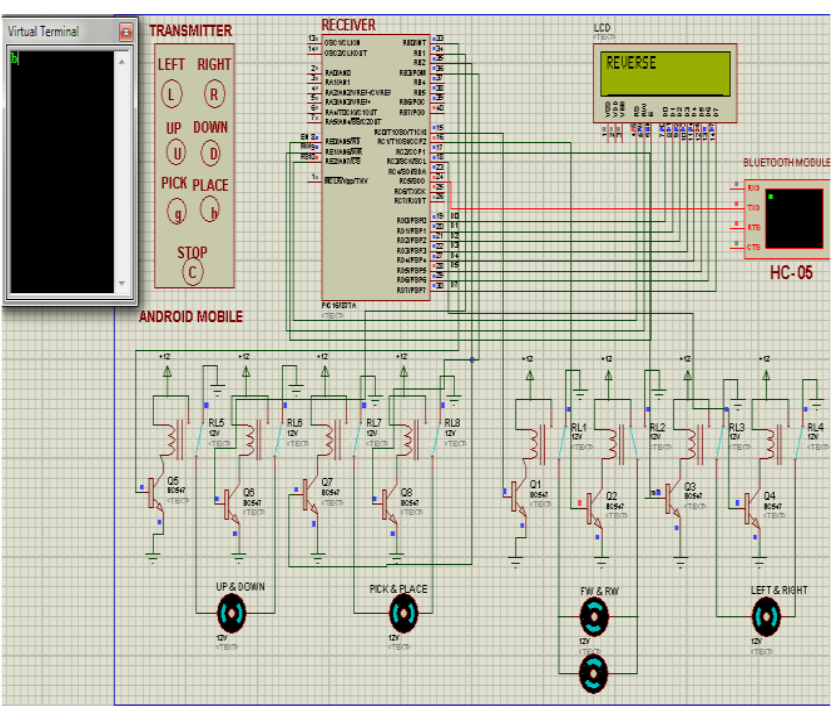

Figure 4.5: Reverse Moving of robot

The button $b$ " which is given in the virtual terminal describes the reverse command where the motor moves in the reverse direction. Forward and reverse motor are connected in parallel manner.

Similarly the following buttons perform the specified functions.

Button _L'- Left Rotation Button $\mathrm{R}^{\prime}$ - Right Rotation Button U'- Upward Moving Button D'- Downward Moving Button P'- Picking the object Button ${ }^{\prime}{ }^{6}$ - Placing the object Button_C'- Stop

\section{Conclusion}

This robot setup reduces the complexity because of wireless connection. The human operator uses android application, so it is easy to access for everyone. This is implemented only for the short distance communication and the execution time is reduced. The merits are it can be more effective for control on the slave setup. The complexity is reduced as we are implementing on wireless communication. The cost is also less as compared to others. The pick and place robot is having the very vast area of applications in domestic and industrial use.

\section{References}

[1] H. Boessenkool, D. Abbink, C. Heemskerk, F. van der Helm, and J. Wildenbeest(2012), -A task-specific analysis of the benefit of haptic shared control during tele-manipulation," IEEE Trans. Haptics, vol. 6, pp. 212.

[2] N. Stefanov, C. Passenberg, A. Peer, and M. Buss(2013), Đesign and evaluation of a haptic computer-assistant for tele-manipulation tasks," IEEE Trans. Human-Mach. Syst., vol. 43, pp. 385-397

[3] Ningbo Yu, Member, IEEE, Kui Wang, Yuan Li, Chang $\mathrm{Xu}$, and Jingtai Liu(2015), -AHaptic Shared Control
Algorithm for Flexible Human Assistance to SemiAutonomous Robots " Fusion Eng. Des., vol. 82, no. $15-24$.

[4] J. Wildenbeest, D. Abbink, C. Heemskerk, F. van der Helm, and H. Boessenkool(2012), The impact of haptic feedback quality on the performance of teleoperated assembly tasks," IEEE Trans. Haptics, vol. 6, pp. 1939-1412.

[5] Franck Mars, Mathieu Deroo, and Jean-Michel Hoc(2014), Analysis of Human-Machine Cooperation When Driving with Different Degrees of Haptic Shared Control" vol. 7, pp 324-333.

[6] Henri Boessenkool, David A. Abbink, Cock J.M. Heemskerk and FransC.T. van der Helm(2013), Haptic Shared Control Improves Tele-Operated Task performance towards Performance in Direct Control" IEEE Trans. Robot. Autom., vol. 5, pp 433-438.

[7] Mark Mulder, David A. Abbink and Erwin R. Boer(2008), The Effect of Haptic guidance on Curve Negotiation Behavior of Young, Experienced Drivers " IEEE International Conference on Systems, Man and Cybernetics vol. 82, pp 804-809

[8] Paul Griffiths and R. Brent Gillespie (2010), -Shared Control Between Human and Machine: Haptic Display of Automation During Manual Control of Vehicle Heading" JSAE Review, vol. 24, pp 65-70.

[9] Haiying Hu, Jiawei Li, Zongwu Xie, Bin Wang(2005), -A Robot Arm/Hand Teleoperation System with Telepresence and Shared Control" International Conference on Advanced Intelligent Mechatronics, vol.7, pp 1312-1317.

[10] Kenneth J. Waldron and Mark R. Cutkosky(2003), Shared Control For Dexterous Telemanipulation With Haptic Feedback" Ergonomics,vol.10, pp180 -186. 